PROCEEDINGS OF THE

AMERICAN MATHEMATICAL SOCIETY

Volume 135, Number 12, December 2007, Pages 3943-3948

S 0002-9939(07)08993-9

Article electronically published on June 20, 2007

\title{
THE COMPLETE ISOMORPHISM CLASS OF AN OPERATOR SPACE
}

\author{
TIMUR OIKHBERG
}

(Communicated by N. Tomczak-Jaegermann)

\begin{abstract}
Suppose $X$ is an infinite-dimensional operator space and $n$ is a positive integer. We prove that for every $C>0$ there exists an operator space $\tilde{X}$ such that the formal identity map $i d: X \rightarrow \tilde{X}$ is a complete isomorphism, $I_{\mathbf{M}_{n}} \otimes i d$ is an isometry, and $d_{c b}(X, \tilde{X})>C$. This provides a non-commutative counterpart to a recent result of W. Johnson and E. Odell.
\end{abstract}

\section{INTRODUCTION}

Recently, W. Johnson and E. Odell 2] solved a problem of V. I. Gurarii by showing that the isomorphism class of any separable infinite-dimensional Banach space has infinite diameter with respect to the Banach-Mazur distance. More precisely, they proved that, for every $C>0$, and for every separable infinitedimensional Banach space $X$, there exists a Banach space $\tilde{X}$ isomorphic to $X$ such that the Banach-Mazur distance between these two spaces exceeds $C$. In this paper, we prove a non-commutative counterpart of this result:

Theorem 1. Suppose $X$ is an infinite-dimensional operator space and $n$ is a positive integer. Then for every $C>0$ there exists an operator space $\tilde{X}$ such that the formal identity map id $: X \rightarrow \tilde{X}$ is a complete isomorphism, $I_{\mathbf{M}_{n}} \otimes i d$ is an isometry, and $d_{c b}(X, \tilde{X})>C$.

Here and below, $E \otimes F$ refers to the minimal (or spatial) tensor product of operator spaces $E$ and $F . \mathbf{M}_{n}$ stands for the space of $n \times n$ matrices, with its usual operator space structure. For the sake of brevity, we often use $\mathbf{M}_{n}(E)$ instead of $\mathbf{M}_{n} \otimes E$.

The proof of Theorem 1 (given in Section 3) relies on the properties of the operator space $\operatorname{MIN}_{n}(X)$, which we explore in Section 2. Throughout the paper, we use freely the standard operator space and Banach space terminology and results. The reader is referred to [1, 8, 9] for operator spaces, and [5] for Banach spaces.

Received by the editors June 28, 2006 and, in revised form, October 31, 2006.

2000 Mathematics Subject Classification. Primary 46L07, 47L25.

Key words and phrases. Exact operator space, complete isomorphism, c.b. distance.

The author was partially supported by the NSF grant DMS-0500957.

(C)2007 American Mathematical Society Reverts to public domain 28 years from publication 


\section{The FUNCTOR $\mathrm{MIN}_{n}$}

Recall that (see [4, 6, 7]), for an operator space $E$ and $n \in \mathbb{N}$, we define an operator space $\operatorname{MIN}_{n}(E)$ to be isometric to $E$ on the Banach space level, and

$$
\|x\|_{\mathcal{K}_{0} \otimes \mathrm{MIN}_{n}(E)}=\sup \left\{\left\|\left(I_{\mathcal{K}_{0}} \otimes u\right) x\right\| \mid u \in C B\left(E, \mathbf{M}_{n}\right),\|u\|_{c b} \leqslant 1\right\}
$$

for $x \in \mathcal{K}_{0} \otimes E\left(\mathcal{K}_{0}\right.$ denotes the space of infinite matrices with finitely many non-zero entries). Letting $\mathcal{I}$ be the closed unit ball of $C B\left(E, \mathbf{M}_{n}\right)$, we can view $\operatorname{MIN}_{n}(E)$ as the image of the map $U$ in $\ell_{\infty}\left(\mathcal{I}, \mathbf{M}_{n}\right)$, where $U: E \rightarrow \ell_{\infty}\left(\mathcal{I}, \mathbf{M}_{n}\right)$ is defined by $U(e)=(u(e))_{u \in \mathcal{I}}$. By a compactness argument, for any finite-dimensional subspace $F$ of $\operatorname{MIN}_{n}(E)$, and every $\varepsilon>0$, there exists $k \in \mathbb{N}$ such that $F$ embeds into $\ell_{\infty}^{k}\left(\mathbf{M}_{n}\right)$ $(1+\varepsilon)$-completely isomorphically. Consequently, $\operatorname{MIN}_{n}(E)$ is 1-exact. Recall that an operator space $X$ is $c$-exact if, for every finite-dimensional subspace $Z \hookrightarrow X$, and every $\varepsilon>0$, there exists $N \in \mathbb{N}$ and a $\tilde{Z} \hookrightarrow \mathbf{M}_{N}$ such that $d_{c b}(Z, \tilde{Z})<c+\varepsilon$. The smallest $c$ satisfying this condition is called the exactness constant of $X$ and is denoted by $\operatorname{ex}(X)$.

In addition to $\mathrm{MIN}_{n}$, in Section 3 we also use the "dual" functor $\mathrm{MAX}_{n}$ (see 4, ,7, for more information). One should note that, for any operator space $E, \operatorname{MIN}_{1}(E)$ and $\operatorname{MAX}_{1}(E)$ are identical to $\operatorname{MIN}(E)$ and $\operatorname{MAX}(E)$, respectively (the "minimal" and "maximal" quantizations).

In [6] we proved:

Lemma 2. Suppose $X$ and $Y$ are operator spaces and $n \in \mathbb{N}$.

(1) If $u \in B(X, Y)$, then

$$
\|u\|_{C B\left(X, \mathrm{MIN}_{n}(Y)\right)}=\left\|I_{\mathbf{M}_{n}} \otimes u\right\|_{B\left(\mathbf{M}_{n}(X), \mathbf{M}_{n}(Y)\right)} .
$$

In particular,

$$
\|u\|_{C B\left(\operatorname{MiN}_{n}(X), \operatorname{MIN}_{n}(Y)\right)}=\left\|I_{\mathbf{M}_{n}} \otimes u\right\|_{B\left(\mathbf{M}_{n}(X), \mathbf{M}_{n}(Y)\right)} .
$$

(2) If $X$ is a subspace of $Y$, then $\operatorname{MIN}_{n}(X)$ is a subspace of $\operatorname{MIN}_{n}(Y)$.

More results concerning $\mathrm{MIN}_{n}$ are needed:

Lemma 3. Suppose $X$ is an operator space and $n, s \in \mathbb{N}$. Then, for any $x \in$ $\mathbf{M}_{s}(X)$,

$$
\|x\|_{\mathbf{M}_{s}\left(\mathrm{MIN}_{n}(X)\right)}=\sup \left\|\left(P \otimes I_{X}\right) x\left(Q \otimes I_{X}\right)\right\|_{\mathbf{M}_{s}(X)},
$$

where the supremum runs over all orthogonal projections $P, Q \in \mathbf{M}_{s}$ of rank not exceeding $n$.

Corollary 4. Suppose $X$ is an operator space, $n, s \in \mathbb{N}$, and $s \geqslant n$. Then, for any $x \in \mathbf{M}_{s}(X)$,

$$
\|x\|_{\mathbf{M}_{s}\left(\operatorname{MIN}_{n}(X)\right)} \geqslant\left(\frac{n}{s}\right)^{2}\|x\|_{\mathbf{M}_{s}(X)} .
$$

Proof of Lemma 3. Clearly, we only need to consider the case of $s>n$. If $P$ and $Q$ are orthogonal projections of rank $\leqslant n$, then, by Ruan's axioms,

$$
\left\|\left(P \otimes I_{X}\right) x\left(Q \otimes I_{X}\right)\right\|_{\mathbf{M}_{s}\left(\operatorname{MIN}_{n}(X)\right)} \leqslant\|x\|_{\mathbf{M}_{s}\left(\operatorname{MIN}_{n}(X)\right)} .
$$

Furthermore, $\left(P \otimes I_{X}\right) x\left(Q \otimes I_{X}\right)$ can be thought of as an element of $\mathbf{M}_{n}(X)$. Thus, by [7],

$$
\begin{aligned}
& \left\|\left(P \otimes I_{X}\right) x\left(Q \otimes I_{X}\right)\right\|_{\mathbf{M}_{s}(X)}=\left\|\left(P \otimes I_{X}\right) x\left(Q \otimes I_{X}\right)\right\|_{\mathbf{M}_{s}\left(\operatorname{MIN}_{n}(X)\right)} ; \\
& \text { hence }\|x\|_{\mathbf{M}_{s}\left(\operatorname{MIN}_{n}(X)\right)} \geqslant \sup \left\|\left(P \otimes I_{X}\right) x\left(Q \otimes I_{X}\right)\right\|_{\mathbf{M}_{s}(X)} .
\end{aligned}
$$


To prove the reverse inequality, it suffices to prove that, whenever $\|x\|_{\mathrm{M}_{s}\left(\operatorname{MIN}_{n}(X)\right)}$ $>1$, there exist orthogonal projections $P$ and $Q$, of rank $\leqslant n$, for which $\left\|\left(P \otimes I_{X}\right) x\left(Q \otimes I_{X}\right)\right\|_{\mathbf{M}_{s}(X)}>1$. To do this, find a complete contraction $u$ : $X \rightarrow \mathbf{M}_{n}$, for which $\|y\|_{\mathbf{M}_{s}\left(\mathbf{M}_{n}\right)}>1$, where $y=\left(I_{\mathbf{M}_{s}} \otimes u\right) x$. Then there exist unit vectors $\xi, \eta \in \ell_{2}^{n}\left(\ell_{2}^{s}\right)$ such that $\langle y \xi, \eta\rangle>1$. Write $\xi=\left(\xi_{i}\right)_{i=1}^{n}$ and $\eta=\left(\eta_{i}\right)_{i=1}^{n}$, with $\xi_{1}, \ldots, \xi_{n}, \eta_{1}, \ldots, \eta_{n} \in \ell_{2}^{s}$. Denote by $P$ and $Q$ the orthogonal projections onto $\operatorname{span}\left[\eta_{i} \mid 1 \leqslant i \leqslant n\right]$ and $\operatorname{span}\left[\xi_{i} \mid 1 \leqslant i \leqslant n\right]$, respectively. Then $\left(Q \otimes I_{X}\right) \xi=\xi$, and $\left(P \otimes I_{X}\right) \eta=\eta$. Therefore,

$$
\begin{aligned}
\left\|\left(P \otimes I_{X}\right) x\left(Q \otimes I_{X}\right)\right\|_{\mathbf{M}_{s}(X)} & \geqslant\left\|\left(I_{\mathbf{M}_{s}} \otimes u\right)\left(P \otimes I_{X}\right) x\left(Q \otimes I_{X}\right)\right\|_{\mathbf{M}_{s}\left(\mathbf{M}_{n}\right)} \\
& =\left\|\left(P \otimes I_{\ell_{2}^{n}}\right) y\left(Q \otimes I_{\ell_{2}^{n}}\right)\right\| \\
& \geqslant\left|\left\langle\left(P \otimes I_{\ell_{2}^{n}}\right) y\left(Q \otimes I_{\ell_{2}^{n}}\right) \xi, \eta\right\rangle\right|=\langle y \xi, \eta\rangle>1,
\end{aligned}
$$

as desired.

Proof of Corollary 4, For $S \subset\{1, \ldots, s\}$, we denote by $P_{S}$ the corresponding basis projection on $\ell_{2}^{s}$. That is, $P_{S} e_{i}=0$ if $i \notin S$, and $P_{S} e_{i}=e_{i}$ if $i \in S\left(e_{1}, \ldots, e_{s}\right.$ is the canonical basis in $\left.\ell_{2}^{s}\right)$. An easy calculation shows that, for any $x \in \mathbf{M}_{s}(X)$,

$$
x=\frac{s^{2}}{n^{2}} \operatorname{Ave}\left(P_{S_{1}} \otimes I_{X}\right) x\left(P_{S_{2}} \otimes I_{X}\right),
$$

where the average is taken over all subsets of $\{1, \ldots, s\}$ of cardinality $n$. By Lemma 3 ,

$$
\|x\|_{\mathbf{M}_{s}\left(\operatorname{MIN}_{n}(X)\right)} \geqslant\left\|\left(P_{S_{1}} \otimes I_{X}\right) x\left(P_{S_{2}} \otimes I_{X}\right)\right\|_{\mathbf{M}_{s}(X)}
$$

for any $S_{1}$ and $S_{2}$ as above. Taken together, the two centered expressions yield the proof.

\section{Proof of the MAIN RESUlts}

To prove Theorem 1, we need to introduce some notation. For an operator space $X$ and $\lambda>0$, we denote by $\lambda X$ the operator space, isomorphic to $X$, and equipped with the norm $\|x\|_{\mathcal{K}_{0} \otimes \lambda X}=\lambda\|x\|_{\mathcal{K}_{0} \otimes X}$ (here, $\left.x \in \mathcal{K}_{0} \otimes X\right)$. For $n \in \mathbb{N}$, set $\alpha_{n}(X)=\left\|i d: \operatorname{MIN}_{n}(X) \rightarrow X\right\|_{c b}$ (note that $\alpha_{n}(X) \in[1, \infty]$ ). We have:

Lemma 5. Suppose $n \in \mathbb{N}$, and suppose an operator space $X$ is $C$-completely isomorphic to $\lambda X \cap \operatorname{MIN}_{n}(X)$ for every $\lambda \in(0,1)$. Then $\alpha_{n}(X) \leqslant C$.

Note that, for a compatible couple $\left(Y_{0}, Y_{1}\right)$ of operator spaces, $Y_{0} \cap Y_{1}$ is defined by assigning to any $y \in \mathcal{K}_{0} \otimes\left(Y_{0} \cap Y_{1}\right)$ the norm

$$
\|y\|_{\mathcal{K}_{0} \otimes\left(Y_{0} \cap Y_{1}\right)}=\max \left\{\|y\|_{\mathcal{K}_{0} \otimes Y_{0}},\|y\|_{\mathcal{K}_{0} \otimes Y_{1}}\right\}
$$

(see Section 2.7 of [9] for more information about interpolation).

Proof of Lemma 5. Suppose, for the sake of contradiction, that $\alpha_{n}(X)>C$. Then there exists $x \in \mathbf{M}_{s}(X)(s>n)$ such that $\|x\|_{\mathbf{M}_{s}\left(\operatorname{MIN}_{n}(X)\right)} \leqslant 1$ and $\|x\|_{\mathbf{M}_{s}(X)}>C$. Pick $\lambda \in\left(0, n^{2} / s^{2}\right)$. We shall show that, for any complete contraction $T: X \rightarrow \tilde{X}$ (here, $\tilde{X}=\lambda X \cap \operatorname{MIN}_{n}(X)$ ), we have

$$
\left\|\left(I_{\mathbf{M}_{s}} \otimes T\right) x\right\|_{\mathbf{M}_{s}(\tilde{X})} \leqslant 1
$$

thus obtaining a contradiction. Indeed, the last inequality implies that

$$
\left\|T^{-1}\right\|_{c b} \geqslant \frac{\|x\|_{\mathbf{M}_{s}(X)}}{\left\|\left(I_{\mathbf{M}_{s}} \otimes T\right) x\right\|_{\mathbf{M}_{s}(\tilde{X})}}>C .
$$


Let $i d: X \rightarrow \tilde{X}$ be the formal identity map. Since $\lambda \in(0,1), I_{\mathbf{M}_{n}} \otimes i d$ : $\mathbf{M}_{n}(X) \rightarrow \mathbf{M}_{n}(\tilde{X})$ is an isometry. Therefore, by Lemma 2 id $: \operatorname{MIN}_{n}(X) \rightarrow$ $\operatorname{MIN}_{n}(\tilde{X})$ is a complete isometry, and

$$
\|T\|_{C B\left(\operatorname{MIN}_{n}(X)\right)}=\|T\|_{C B\left(\operatorname{MIN}_{n}(X), \operatorname{MIN}_{n}(\tilde{X})\right)}=\left\|I_{\mathbf{M}_{n}} \otimes T\right\| \leqslant\|T\|_{C B(X, \tilde{X})} \leqslant 1,
$$

and $\left\|\left(I_{\mathbf{M}_{s}} \otimes T\right) x\right\|_{\mathbf{M}_{s}\left(\operatorname{MIN}_{n}(X)\right)} \leqslant 1$.

Now recall that

$$
\begin{aligned}
& \left\|\left(I_{\mathbf{M}_{s}} \otimes T\right) x\right\|_{\mathbf{M}_{s}(\tilde{X})} \\
& =\max \left\{\lambda\left\|\left(I_{\mathbf{M}_{s}} \otimes T\right) x\right\|_{\mathbf{M}_{s}(X)},\left\|\left(I_{\mathbf{M}_{s}} \otimes T\right) x\right\|_{\mathbf{M}_{s}\left(\operatorname{MIN}_{n}(X)\right)}\right\} .
\end{aligned}
$$

By (3.2), $\left\|\left(I_{\mathbf{M}_{s}} \otimes T\right) x\right\|_{\mathbf{M}_{s}\left(\operatorname{MIN}_{n}(X)\right)} \leqslant 1$. Moreover, by Corollary 4 .

$$
\left\|\left(I_{\mathbf{M}_{s}} \otimes T\right) x\right\|_{\mathbf{M}_{s}(X)} \leqslant \frac{s^{2}}{n^{2}}\left\|\left(I_{\mathbf{M}_{s}} \otimes T\right) x\right\|_{\mathbf{M}_{s}\left(\operatorname{MIN}_{n}(X)\right)} \leqslant \frac{s^{2}}{n^{2}} .
$$

Therefore, by (3.3), and by the choice of $\lambda$,

$$
\left\|\left(I_{\mathbf{M}_{s}} \otimes T\right) x\right\|_{\mathbf{M}_{s}(\tilde{X})} \leqslant \max \left\{\frac{\lambda s^{2}}{n^{2}}, 1\right\} \leqslant 1,
$$

which yields (3.1).

Now, we are ready to establish the main result.

Proof of Theorem 1. Suppose, for the sake of contradiction, that $X$ is an infinitedimensional operator space, and there exists $C>0$ such that $d_{c b}(X, \tilde{X}) \leqslant C$ whenever $\tilde{X}$ is completely isomorphic to $X$, and $I_{\mathbf{M}_{n}} \otimes i d: \mathbf{M}_{n}(X) \rightarrow \mathbf{M}_{n}(\tilde{X})$ is an isometry. As noted in the proof of Lemma [5, the formal identity $i d: X \rightarrow$ $\lambda X \cap \operatorname{MIN}_{n}(X)(\lambda \in(0,1))$ is an isometry, and moreover, $I_{\mathbf{M}_{n}} \otimes i d$ is an isometry. Furthermore,

$$
\|i d\|_{C B\left(X, \lambda X \cap \mathrm{MIN}_{n}(X)\right)}=\max \left\{\|i d\|_{C B(X, \lambda X)},\|i d\|_{C B\left(X, \mathrm{MIN}_{n}(X)\right)}\right\} \leqslant 1
$$

and

$$
\left\|i d^{-1}\right\|_{C B\left(\lambda X \cap \mathrm{MIN}_{n}(X), X\right)} \leqslant\left\|i d^{-1}\right\|_{C B(\lambda X, X)}=\lambda^{-1} .
$$

Thus, $X$ is completely isomorphic to $\lambda X \cap \operatorname{MIN}_{n}(X)$. If $d_{c b}\left(X, \lambda X \cap \operatorname{MIN}_{n}(X)\right) \leqslant C$ for any $\lambda \in(0,1)$, then, by Lemma $5, \alpha_{n}(X) \leqslant C$. Therefore, ex $(X) \leqslant C$.

Now consider the space $\tilde{X}=\lambda^{-1} X+\operatorname{MAX}_{n}(X)$ (as before, $\lambda \in(0,1)$ ). Denoting, once again, by $i d$ the formal identity map from $X$ to $\tilde{X}$, we see that $I_{\mathbf{M}_{n}} \otimes i d$ is an isometry (that is, $\|x\|_{\mathbf{M}_{n}(X)}=\|x\|_{\mathbf{M}_{n}(\tilde{X})}$ for any $x \in \mathbf{M}_{n}(X)$ ), $i d^{-1}$ is a complete contraction, and $\left.\|i d\| \leqslant \lambda^{-1}\right)$. If $d_{c b}(\tilde{X}, X) \leqslant C$, then $d_{c b}\left(\tilde{X}^{*}, X^{*}\right) \leqslant C$. However, by 7 ,

$$
\tilde{X}^{*}=\left(\lambda^{-1} X\right)^{*} \cap\left(\operatorname{MAX}_{n}(X)\right)^{*}=\lambda X^{*} \cap \operatorname{MIN}_{n}\left(X^{*}\right) .
$$

Thus, by Lemma 5, $\alpha_{n}\left(X^{*}\right) \leqslant C$; hence $\operatorname{ex}\left(X^{*}\right) \leqslant C$.

The exactness of both $X$ and $X^{*}$ implies, by [1] (see also [10]), that $X$ is completely isomorphic to $H^{r} \oplus K^{c}$, where $H^{r}$ and $K^{c}$ denote the Hilbert spaces $H$ and $K$ equipped with their column, resp. row, operator space structures. In particular, there exists a constant $c>0$ such that $(1) \operatorname{ex}(X) \leqslant c$, and (2) $X$ contains $c$-completely complemented subspaces of arbitrarily large dimension (in particular, those arising from $H_{0}^{r} \oplus K_{0}^{c}$, where $H_{0}$ and $K_{0}$ are subspaces of $H$ and $K$, respectively). 
It remains to construct an operator space $\tilde{X}$, completely isomorphic to $X$, and such that $I_{\mathbf{M}_{n}} \otimes i d$ is an isometry, yet $\operatorname{ex}(\tilde{X})>C c$. Then the inequality $d_{c b}(X, \tilde{X}) \geqslant$ $\operatorname{ex}(\tilde{X}) / \operatorname{ex}(X)>C$ will deliver the desired contradiction.

To this end, pick a subspace $E$ of $X$ such that $\left(4 C c^{2} n^{2}\right)^{2}<N=\operatorname{dim} E<\infty$ and such that there exists a projection $P$ from $X$ onto $E$ with $\|P\|_{c b} \leqslant c$. Let $j: E \rightarrow \operatorname{MAX}(E)$ be the formal identity map, and consider $u=\left(c n^{2}\right)^{-1} j P \in$ $C B(X, \operatorname{MAX}(E))$. Define the operator space $\tilde{X}$ by setting, for $x \in \mathcal{K}_{0} \otimes X$,

$$
\|x\|_{\mathcal{K}_{0} \otimes \tilde{X}}=\max \left\{\|x\|_{\mathcal{K}_{0} \otimes X},\left\|\left(I_{\mathcal{K}_{0}} \otimes u\right) x\right\|_{\mathcal{K}_{0} \otimes \operatorname{MAX}(E)}\right\} .
$$

Denoting by $i d$ the formal identity map from $X$ to $\tilde{X}$, we observe that

$$
\|i d\|_{c b} \leqslant\|u\|_{c b} \leqslant\left(c n^{2}\right)^{-1}\|j\|_{c b}\|P\|_{c b} \leqslant N / n^{2}<\infty
$$

(here, we use the fact that $\|j\|_{c b} \leqslant N$; see, e.g., Chapter 3 of [9], or [12] for a better estimate). Moreover, $\|u\| \leqslant\left(c n^{2}\right)^{-1}\|P\| \leqslant n^{-2}$. By Corollary 4 (and the fact that, for every operator space $\left.Y, \operatorname{MIN}(Y)=\operatorname{MIN}_{1}(Y)\right),\|e\|_{\mathbf{M}_{n}(\operatorname{MAX}(E))} \leqslant$ $n^{2}\|e\|_{\mathbf{M}_{n}(\operatorname{MIN}(E))}$ for any $e \in \mathbf{M}_{n}(E)$. Therefore,

$$
\left\|I_{\mathbf{M}_{n}} \otimes u\right\| \leqslant n^{2}\left\|I_{\mathbf{M}_{n}} \otimes u\right\|_{B\left(\mathbf{M}_{n}(X), \mathbf{M}_{n}(\operatorname{MiN}(E))\right)}=n^{2}\|u\|=1 .
$$

Thus, $I_{\mathbf{M}_{n}} \otimes i d$ is an isometry.

It remains to estimate $\operatorname{ex}(\tilde{X})$ from below. Denote by $\tilde{E}$ the image of $E$ in $\tilde{X}$, and by $\tilde{j}$ the formal identity map from $\tilde{E}$ to $\operatorname{MAX}(E)$. Then $\left\|\tilde{j}^{-1}\right\|_{c b}=\left\|\tilde{j}^{-1}\right\| \leqslant 1$. Moreover,

$$
\|e\|_{\mathbf{M}_{s}(\tilde{E})} \geqslant\left\|\left(I_{\mathbf{M}_{s}} \otimes u\right) e\right\|_{\mathbf{M}_{s}(\operatorname{MAX}(E))}=\left(c n^{2}\right)^{-1}\left\|\left(I_{\mathbf{M}_{s}} \otimes j\right) e\right\|_{\mathbf{M}_{s}(\operatorname{MAX}(E))}
$$

for any $e \in \mathbf{M}_{s}(\tilde{E})$; hence $\|\tilde{j}\|_{c b} \leqslant c n^{2}$. Therefore,

$$
\operatorname{ex}(\tilde{X}) \geqslant \operatorname{ex}(\tilde{E}) \geqslant \frac{\operatorname{ex}(\operatorname{MAX}(E))}{\|\tilde{j}\|_{c b}\left\|\tilde{j}^{-1}\right\|_{c b}} \geqslant \frac{\sqrt{N}}{4 c n^{2}}>C c
$$

(by [3], $\operatorname{ex}(\operatorname{MAX}(E)) \geqslant \sqrt{N} / 4)$.

\section{ACKNOWLEDGMENTS}

We would like to thank the organizers of of the workshop in Linear Analysis and Probability in College Station, Texas, in 2004 and 2005, where part of this work was carried out.

\section{REFERENCES}

1. E. Effros and Z.-J. Ruan, Operator spaces, Oxford University Press, New York, 2000. MR 1793753 (2002a:46082)

2. W. Johnson and E. Odell, The diameter of the isomorphism class of a Banach space, Ann. Math. 162 (2005), 423-437. MR2178965 (2006j:46011)

3. M. Junge and G. Pisier, Bilinear forms on exact operator spaces and $B(H) \otimes B(H)$, Geom. Funct. Anal. 5 (1995), 329-363. MR 1334870 (96i:46071)

4. F. Lehner, $M_{n}$ espaces, sommes d'unitaires et analyse harmonique sur le groupe libre, $\mathrm{Ph} . \mathrm{D}$. thesis, Université Paris VI, 1997.

5. J. Lindenstrauss and L. Tzafriri, Classical Banach spaces I, Springer-Verlag, Berlin, 1977. MR0500056 (58:17766)

6. T. Oikhberg, The non-commutative Gurarii space, Arch. Math. 86 (2006), 356-364. MR 2223271

7. T. Oikhberg and E. Ricard, Operator spaces with few completely bounded maps, Math. Ann. 328 (2004), 229-259. MR2030376 (2005g:46113) 
8. V. Paulsen, Completely bounded maps and operator algebras, Cambridge University Press, 2002. MR 1976867 (2004c:46118)

9. G. Pisier, An introduction to the theory of operator spaces, Cambridge University Press, 2003. MR 2006539 (2004k:46097)

10. G. Pisier, Completely bounded maps into certain Hilbertian operator spaces, Int. Math. Res. Not. 74 (2004), 3983-4018. MR2103799 (2005g:46114)

11. G. Pisier and D. Shlyakhtenko, Grothendieck's theorem for operator spaces, Invent. Math. 150 (2002), 185-217. MR1930886 (2004k:46096)

12. E. Ricard, Décompositions de $H^{1}$, multplicateurs de Schur et espaces d'opérateurs, Ph.D. thesis, Université Paris VI, 2001.

Department of Mathematics, University of California, Irvine, Irvine, California 92697

E-mail address: toikhber@math.uci.edu 